\title{
An Algorithm for the Zeros of Transcendental Functions
}

\section{Soon Park Chung}

Department of Mathematics, University of Michigan-Dearborn, Dearborn, Michigan 48128, USA

Summary. In this paper, we extend the dual form of the generalized algorithm of Sebastião e Silva [3] for polynomial zeros and show that it is effective for finding zeros of transcendental functions in a circle of analyticity.

Subject Classifications. AMS(MOS): 65H05 R: 5.15.

\section{§1. Introduction}

Let $f(z)$ be a polynomial of degree $n$. For convenience, $f(z)$ will be taken as normalized:

$$
\begin{aligned}
f(z) & =1+a_{1} z+a_{2} z^{2}+\cdots+a_{n} z^{n} \\
& =\left(1-r_{1}^{-1} z\right)\left(1-r_{2}^{-1} z\right) \cdots\left(1-r_{n}^{-1} z\right) .
\end{aligned}
$$

For any function $p(z)$ such that $p(0) \neq 0$, it will be understood that

$$
p^{+}(z)=p(z) / p(0),
$$

throughout this paper. We will now state a global method for finding the zeros $r_{i}$ of $f(z)$.

Theorem 1.1. Let $f(z)$ be given by (1.1). Let $g(z)$ and $g_{0}(z)$ be any polynomials of degree $n-1$ at most such that neither $g(z), g^{\prime}(z)$ nor $g_{0}(z)$ vanishes for any $r_{i}$, and $g\left(r_{i}\right) \neq g\left(r_{j}\right)$ for $r_{i} \neq r_{j}$. Let $k$ be the degree of $g(z)$ and define recursively

$$
g_{v+1}(z)=[g(z)]^{-1}\left[g_{v}(z)-\phi_{v}(z) f(z)\right], \quad v=0,1,2, \ldots,
$$

where each $\phi_{v}(z)$ is of degree $k-1$ at most such that $g_{v}(z)-\phi_{v}(z) f(z)$ is divisible by $\mathrm{g}(\mathrm{z})$.

$$
\begin{aligned}
& \text { If } \\
& \left|g\left(r_{1}\right)\right|<\left|g\left(r_{2}\right)\right| \leqq \cdots \leqq\left|g\left(r_{n}\right)\right|
\end{aligned}
$$


then

$$
\lim _{v \rightarrow \infty} g_{v}^{+}(z)=f(z) /\left(1-r_{1}^{-1} z\right) .
$$

Moreover, let

$$
g_{v, 1}(z)=g_{v}(z), \quad \text { for each } v,
$$

and define the sequences $g_{v, p}(z), p=1,2, \ldots, n$, by either of the following rules:

Rule 1: Form $g_{v, p+1}(z)$ by eliminating the constant term between $g_{v, p}(z)$ and $g_{v+1, p}(z)$ and dividing by $z$.

Rule 2: Define $g_{v, p}(z)$ as the following determinant of order $p$ :

$$
g_{v, p}(z)=\delta\left(\begin{array}{ccc}
g_{v}(z) & g_{v+1}(z) \ldots & g_{v+p-1}(z) \\
g_{v+1}(z) & g_{v+2}(z) \ldots & g_{v+p}(z) \\
\ldots \ldots \ldots \ldots \ldots \ldots \ldots \ldots \ldots \ldots \ldots \ldots \\
g_{v+p-1}(z) & g_{v+p}(z) \ldots g_{v+2 p-2}(z)
\end{array}\right) \cdot[-f(z)]^{-(p-1)} .
$$

Then

$$
\lim _{v \rightarrow \infty} g_{v, p}^{+}(z)=f(z) /\left[\left(1-r_{1}^{-1} z\right) \ldots\left(1-r_{p}^{-1} z\right)\right]
$$

if

$$
\left|g\left(r_{1}\right)\right| \leqq \cdots \leqq\left|g\left(r_{p}\right)\right|<\left|g\left(r_{p+1}\right)\right| \leqq \cdots \leqq\left|g\left(r_{n}\right)\right| .
$$

The algorithm presented above is in the dual form. The direct form of the algorithm has the recursion

$$
g_{v+1}(z)=g(z) g_{v}(z)-\alpha_{v}(z) f(z), \quad v=0,1,2, \ldots,
$$

where each $g_{v}(z)$ is of degree $n-1$ at most, in place of the recursion (1.3).

The discoverer of the idea of this algorithm is Sebastião e Silva [7]. He uses

$$
g(z)=z, \quad g_{0}(z)=1,
$$

and defines $g_{v, p}(z)$ in a little different way. His method has been further elaborated by Bauer $[1,2]$, who gives treppeniteration for forming the sequences $g_{v, p}(z)$; and generalized by Householder [5], who has shown that an almost arbitrary polynomial can be used for $g(z)$.

Chung [3] has added an elimination rule, Rule 1, for defining the sequences $\mathbf{g}_{v, p}(z)$ and introduced the accelerated forms of the algorithm which are quadratically convergent.

In this paper we will show that this algorithm can be extended to adapt to transcendental functions in a circle of analyticity. Stewart [8] has shown this for the case $g(z)=z$. The advantages of the general algorithm where $g(z)$ can be any polynomial satisfying some mild conditions are that the zeros of equal modulus can also be handled and that an acceleration in convergence can be obtained by a proper choice of $g(z)$. 
The transcendental function $f(z)$ to be considered in this paper will be taken as normalized, for convenience, i.e., $f(z)$ has the series expansion

$$
f(z)=1+a_{1} z+a_{2} z^{2}+\cdots .
$$

Let $f(z)$ be analytic in a circle

$$
C_{R}=\{z|| z \mid<R\},
$$

where $R>0$ is a fixed real number. Also, let $f(z)$ have exactly $n$ zeros, $r_{1}, r_{2}, \ldots, r_{n}$, in $C_{R}$ counting multiplicities. Define

$$
\Pi^{\langle p\rangle}(z)=\left(1-r_{1}^{-1} z\right)\left(1-r_{2}^{-1} z\right) \ldots\left(1-r_{p}^{-1} z\right),
$$

and

$$
f^{\langle p\rangle}(z)=f(z) / \Pi^{\langle p\rangle}(z),
$$

for $p=1,2, \ldots, n$. Also, define

$$
G_{1}^{P}=\left\{v(z) \cdot f^{\langle p\rangle}(z) \mid v(z) \text { is a polynomial of degree } p-1 \text { at most }\right\}
$$

and

$$
G_{2}^{P}=\left\{u(z) \Pi^{\langle p\rangle}(z) \mid u(z) \in G\right\},
$$

where

$$
G=\left\{q(z) \mid q(z) \text { is an analytic function in } C_{R}\right\} .
$$

We have the following lemma which is due to Stewart [8].

Lemma 1.1. Let $r_{i} \notin\left\{r_{1}, r_{2}, \ldots, r_{p}\right\}$ for $i=p+1, p+2, \ldots, n$. Then for any $q(z) \in G$, there exist $q_{1}(z) \in G_{1}^{P}$ and $q_{2}(z) \in G_{2}^{P}$ such that

$$
q(z)=q_{1}(z)+q_{2}(z) .
$$

Moreover, this decomposition is unique.

In $\S 2$, we will introduce a class of generalized operators which produce the basic sequence $g_{\mathrm{v}}(z)$. Many of the ideas in this section are taken from Stewart's work mentioned above. In $\S 3$, we will give the algorithm and in $\$ 4$ numerical results will be presented.

\section{§2. The Generalized Operators}

Let $g(z)$ be a polynomial which does not have zeros in common with $f(z)$ and whose zeros are in $C_{R}$. Let $k$ be the degree of $g(z)$ and define an operator $F_{g}: G \rightarrow G$ by

$$
F_{g}(p(z))=[1 / g(z)][p(z)-\phi(z) f(z)],
$$


where $\phi(z)$ is a polynomial of degree $k-1$ at most that is uniquely determined by

$$
\left.\frac{d^{j}(\phi f)}{d z^{j}}\right|_{z=\alpha_{i}}=\left.\frac{d^{j} p}{d z^{j}}\right|_{z=\alpha_{i}}, \quad j=0,1,2, \ldots, m_{i}-1,
$$

for any zero $\alpha_{i}$ of $g(z)$ of multiplicity $m_{i}$. Also, let

$$
F_{g}^{v}(p(z))=F_{g}\left(F_{g}^{v-1}(p(z))\right), \quad v=1,2, \ldots,
$$

and

$$
F_{g}^{0}(p(z))=p(z) .
$$

The properties of $F_{g}^{v}$ follow from the following lemma which is easily proved by induction.

Lemma 2.1. Let $g_{0}(z) \in G$. Assume that $r_{i} \notin\left\{r_{1}, r_{2}, \ldots, r_{p}\right\}$ for $i=p+1, p+2, \ldots, n$. Let

$$
g_{0}(z)=p_{0}(z)+q_{0}(z)
$$

where $p_{0}(z) \in G_{1}^{p}$ and $q_{0}(z) \in G_{2}^{p}$. For $v=1,2, \ldots$, let

$$
\begin{aligned}
& g_{v}(z)=F_{g}^{v}\left(g_{0}(z)\right), \\
& p_{v}(z)=F_{g}^{v}\left(p_{0}(z)\right),
\end{aligned}
$$

and

$$
q_{v}(z)=F_{g}^{v}\left(q_{0}(z)\right) .
$$

Then

$$
p_{v}(z) \in G_{1}^{p}, \quad q_{\nu}(z) \in G_{2}^{p}, \quad v=1,2, \ldots
$$

Moreover,

$$
g_{v}(z)=p_{v}(z)+q_{v}(z), \quad v=1,2, \ldots
$$

For any real number $\rho>0$, let

$$
\begin{aligned}
& \Omega_{\rho}=\{z|| g(z) \mid<\rho\}, \\
& \bar{\Omega}_{\rho}=\{z|| g(z) \mid \leqq \rho\},
\end{aligned}
$$

and

$$
\gamma_{\rho}=\{z|| g(z) \mid=\rho\},
$$

throughout this paper.

Lemma 2.2. Let $\mathrm{g}_{0} \in G_{2}^{p}$ and define the sequence $\mathrm{g}_{v}(z)$ by (2.3). If

$$
\left|g\left(r_{1}\right)\right| \leqq\left|g\left(r_{2}\right)\right| \leqq \cdots \leqq\left|g\left(r_{p}\right)\right|<\left|g\left(r_{p+1}\right)\right| \leqq \cdots \leqq\left|g\left(r_{n}\right)\right|,
$$


then for any real number $\rho$ such that

$$
0<\rho<\left|g\left(r_{p+1}\right)\right|, \bar{\Omega}_{\rho} \subset C_{R},
$$

if such a $\rho$ exists, we can find a constant $M_{z}$ depending upon $\rho$ and $z$ such that

$$
\left|g_{v}(z)\right| \leqq M_{z} \rho^{-v}, \quad \text { for } z \in \Omega_{\rho} .
$$

Moreover, for any $\rho_{0}$ such that $0<\rho_{0}<\rho$, we can find $M_{\rho_{0}}$ depending upon $\rho$ and $\rho_{0}$ such that

$$
\left|g_{v}(z)\right| \leqq M_{\rho_{0}} \rho^{-v}, \quad \text { for all } z \in \bar{\Omega}_{\rho_{0}} .
$$

Proof. Let $\rho$ be a real number satisfying (2.9) and let

$$
q_{v}(z)=g_{v}(z) / f(z), \quad v=0,1,2, \ldots .
$$

It is easy to see that each $q_{v}(z)$ is analytic in $\bar{\Omega}_{\rho}$.

Since, for each $v$,

$$
g_{v+1}(z)=F_{g}\left(g_{v}(z)\right),
$$

we have

$$
g_{v+1}(z)=[1 / g(z)]\left[g_{v}(z)-\phi_{v}(z) f(z)\right],
$$

where $\phi_{v}$ is a polynomial determined as in (2.2), and by dividing (2.12) by $f(z)$, we obtain

$$
q_{v}(z)=\phi_{v}(z)+g(z) q_{v+1}(z) .
$$

Hence, we can see that, for $v=1,2, \ldots$,

$$
q_{0}(z)=\left[\sum_{n=0}^{v-1} \phi_{n}(z) g^{n}(z)\right]+q_{v}(z) g^{v}(z),
$$

and

$$
q_{v}(z)=q_{0}(z) / g^{v}(z)-\sum_{n=0}^{v-1} \phi_{n}(z) / g^{v-n}(z) .
$$

By Cauchy's integral formula, we have, for $z \in \Omega_{\rho}$,

$$
\begin{aligned}
q_{v}(z)= & (2 \pi i)^{-1} \int_{\gamma_{\rho}} q_{\nu}(\xi) d \xi /(\xi-z) \\
= & (2 \pi i)^{-1} \int_{\gamma_{\rho}} q_{0}(\xi) d \xi /\left[g^{v}(\xi)(\xi-z)\right] \\
& -(2 \pi i)^{-1} \sum_{n=0}^{\nu-1} \int_{\gamma_{\rho}} \phi_{n}(\xi) d \xi /\left[g^{v-n}(\xi)(\xi-z)\right] .
\end{aligned}
$$

We now wish to represent $\phi_{n}(\xi) /\left[g^{v-n}(\xi)(\xi-z)\right]$ as a sum of partial fractions. In the simplest case the zeros $\alpha_{i}, i=1,2, \ldots, k$, of $g(z)$ are distinct and $z$ is not equal 
to any $\alpha_{i}$. Then the representation is

$$
\left[\sum_{i=1}^{k} \sum_{j=1}^{v-n} c_{i j} /\left(\xi-\alpha_{i}\right)^{j}\right]+c /(\xi-z)
$$

In the other cases, some minor changes occur in the representation, which do not affect our discussion below. In (2.13), it can be seen that $\left(\sum_{i=1}^{k} c_{i 1}\right)+c$ is the coefficient of $\xi^{k(v-n)}$ in the polynomial $\phi_{n}(\xi)$ of degree $k-1$ at most and hence must be zero for $n=0,1,2, \ldots, v-1$. Since $\gamma_{\rho}$ is a lemniscate [9], we have

$$
\int_{\gamma_{\rho}} d \xi /(\xi-z)=2 \pi i, z \in \Omega_{\rho} .
$$

So, for all $z \in \Omega_{\rho}$,

$$
\int_{\gamma_{\rho}} \phi_{n}(\xi) d \xi /\left(g^{v-n}(\xi)(\xi-z)\right)=2 \pi i\left(\left(\sum_{i=1}^{k} c_{i 1}\right)+c\right)=0,
$$

for $n=0,1,2, \ldots, v-1$, and hence

$$
\begin{aligned}
\left|q_{v}(z)\right| & =\left|(2 \pi i)^{-1} \int_{\gamma_{\rho}} q_{0}(\xi) d \xi /\left[g^{v}(\xi)(\xi-z)\right]\right| \\
& \leqq N \rho^{-v}(2 \pi)^{-1} \int_{\gamma_{\rho}}|d \xi| /|\xi-z|
\end{aligned}
$$

where

Let

$$
N=\max _{\xi \in \gamma_{\rho}}\left|q_{0}(\xi)\right| .
$$

$$
M_{z}=|f(z)| N(2 \pi)^{-1} \int_{\gamma_{\rho}}|d \xi| /|\xi-z| .
$$

Then, for each $v$,

$$
\left|g_{v}(z)\right|=\left|q_{v}(z)\right| \cdot|f(z)| \leqq M_{z} \rho^{-v}, \quad \text { for } z \in \Omega_{\rho} .
$$

Moreover, if we let

$$
M_{\rho_{0}}=N \cdot \max _{z \in \bar{\Omega} \rho_{0}}|f(z)| \cdot\left(2 \pi \min _{z \in \bar{\Omega} \rho_{0}}|\xi-z|\right)^{-1} \int_{\gamma_{\rho}}|d \xi|,
$$

for $0<\rho_{0}<\rho$, then for each $v,(2.11)$ holds, and the proof is complete.

Lemma 2.2, together with Cauchy's integral formula for the derivatives, leads to the following lemma.

Lemma 2.3. Let $g_{0} \in G_{2}^{p}$ and define $g_{v}(z)$ by (2.3). Let $g_{v}(z)$ have the series expansion

$$
\mathrm{g}_{v}(z)=b_{v_{0}}+b_{v_{1}} z+b_{v_{2}} z^{2}+\cdots, \quad v=0,1,2, \ldots .
$$

Let (2.8) hold and let

$$
|g(0)|<\left|g\left(r_{p+1}\right)\right| .
$$


Then, for any real number $\rho$ such that

$$
|g(0)|<\rho<\left|g\left(r_{p+1}\right)\right|, \quad \bar{\Omega}_{\rho} \subset C_{R},
$$

if such a $\rho$ exists, we can find a constant $K$ depending upon $\rho$ such that

$$
\left|b_{v_{j}}\right| \leqq K \rho^{-v}, \quad \text { for all } v \text { and } j=0,1,2, \ldots, p-1 .
$$

\section{§3. The Algorithm}

In the following two theorems, we will show that Theorem 1.1 can be extended to adapt to transcendental functions with a few restrictions on the operating polynomial $g(z)$.

Theorem 3.1. Let $\mathrm{g}(z)$ be a polynomial whose zeros are in $C_{R}$ and which satiesfies all the conditions described in Theorem 1.1. Let $g_{0}(z) \in G$ and $g_{0}\left(r_{i}\right) \neq 0$ for $i=$ $1,2, \ldots, n$. Define the sequence $g_{v}(z)$ by (2.3). If

$$
\left|g\left(r_{1}\right)\right|<\left|g\left(r_{2}\right)\right| \leqq \cdots \leqq\left|g\left(r_{n}\right)\right|
$$

and if there exists a real number $\rho>0$ such that

$$
\left|g\left(r_{1}\right)\right|<\rho<\left|g\left(r_{2}\right)\right| \text { and } \bar{\Omega}_{\rho} \subset C_{R}
$$

then

$$
\lim _{v \rightarrow \infty} g_{v}^{+}(z)=f^{\langle 1\rangle}(z) \quad \text { for } z \in \Omega_{\rho} .
$$

Proof. By Lemma 1.1, there exist $p_{0}(z) \in G_{1}^{1}$ and $q_{0}(z) \in G_{2}^{1}$ such that

$$
g_{0}(z)=p_{0}(z)+q_{0}(z) \text {. }
$$

\section{Let}

$$
p_{v}(z)=F_{g}^{v}\left(p_{0}(z)\right), \quad q_{v}(z)=F_{g}^{v}\left(q_{0}(z)\right), \quad v=1,2, \ldots
$$

Then, by Lemma 2.1, for each $v$,

$$
g_{v}(z)=p_{v}(z)+q_{v}(z) ; \quad p_{v}(z) \in G_{1}^{1}, q_{v}(z) \in G_{2}^{1} .
$$

By Lemma 2.2 there exists a constant $M_{z}$ such that

$$
\left|q_{v}(z)\right| \leqq M_{z} \rho^{-v}, z \in \Omega_{\rho} .
$$

Now, for each $v$, we have

$$
p_{v}(z)=v_{v} f^{\langle 1\rangle}(z),
$$

where $v_{v}$ is a constant, and

$$
v_{v+1}=[1 / g(z)]\left[v_{v}-\phi_{v}(z) \Pi^{\langle 1\rangle}\right], \quad v=0,1,2, \ldots
$$


So

$$
v_{v}=v_{0} / g^{v}\left(r_{1}\right), \quad v=0,1,2, \ldots
$$

Since $g_{0}\left(r_{1}\right) \neq 0$ and $q_{0}\left(r_{1}\right)=0$, we have $p_{0}\left(r_{1}\right) \neq 0$. So $v_{0} \neq 0$ and hence

$$
v_{\mathrm{v}} \neq 0, \quad v=0,1,2, \ldots .
$$

From (3.3), (3.4) and (3.5), we have

$$
\left|q_{v}(z)\right| /\left|p_{v}(z)\right| \leqq M_{z} \rho^{-v}\left|v_{0}\right|^{-1}\left|g\left(r_{1}\right)\right|^{v} /\left|f^{\langle 1\rangle}(z)\right|, \quad v=0,1,2, \ldots,
$$

for each $z \in \Omega_{\rho}$. Since $\rho>\left|g\left(r_{1}\right)\right|$, we have

$$
\lim _{v \rightarrow \infty}\left|q_{v}(z)\right| /\left|p_{v}(z)\right|=0,
$$

and hence

$$
\lim _{v \rightarrow \infty} g_{v}(z) / p_{v}(z)=1,
$$

for each $z \in \Omega_{\rho}$. Moreover, from (3.4) and (3.6),

$$
\lim _{v \rightarrow \infty} p_{v}^{+}(z)=f^{\langle 1\rangle}(z) .
$$

Hence, by (3.7),

$$
\lim _{\nu \rightarrow \infty} g_{\nu}^{+}(z)=f^{\langle 1\rangle}(z), z \in \Omega_{\rho} .
$$

Theorem 3.2. Let $g_{0}(z)$ and $g(z)$ satisfy all the conditions described in Theorem 3.1. Define $g_{v}(z)$ by (2.3). Let

$g_{v, 1}(z)=g_{v}(z)$, for each $v$.

(I) Define the sequences $g_{v, p}(z), p=2, \ldots, n$, by Rule 1 of Theorem 1.1. If (2.8) and (2.14) hold and if there exists a real number $\rho>0$ such that

$$
\left|g\left(r_{p}\right)\right|<\rho<\left|g\left(r_{p+1}\right)\right|, \quad|g(0)|<\rho \text { and } \bar{\Omega}_{\rho} \subset C_{R},
$$

then

$$
\lim _{v \rightarrow \infty} g_{v, p}^{+}(z)=f^{\langle p\rangle}(z) \quad \text { for } z \in \Omega_{\rho} .
$$

(II) Define the sequences $g_{v, p}(z), p=2, \ldots, n$, by Rule 2 of Theorem 1.1. If (2.8) holds, and if there exists a real number $\rho>0$ such that

$$
\left|g\left(r_{p}\right)\right|<\rho<\left|g\left(r_{p+1}\right)\right|, \bar{\Omega}_{\rho} \subset C_{R},
$$

then

$$
\lim _{v \rightarrow \infty} g_{v, p}^{+}(z)=f^{\langle p\rangle}(z) \quad \text { for } z \in \Omega_{\rho} .
$$


Proof. We will prove the second part of the theorem. Let

$$
g_{0}(z)=p_{0}(z)+q_{0}(z)
$$

where $p_{0}(z) \in G_{1}^{p}$ and $q_{0}(z) \in G_{2}^{p}$. Then,

$$
p_{0}\left(r_{i}\right) \neq 0, \quad i=1,2, \ldots, p,
$$

since $g_{0}\left(r_{i}\right) \neq 0$ while $q_{0}\left(r_{i}\right)=0$.

Let

$$
p_{v}(z)=F_{g}^{v}\left(p_{0}(z)\right), \quad q_{v}(z)=F_{g}^{v}\left(q_{0}(z)\right), \quad v=0,1,2, \ldots .
$$

Then, by Lemma 2.1, for each $v$,

$$
g_{v}(z)=p_{v}(z)+q_{v}(z) ; \quad p_{v}(z) \in G_{1}^{p}, q_{v}(z) \in G_{2}^{p} .
$$

Let

$$
p_{v}(z)=v_{v}(z) f^{\langle p\rangle}(z), \quad v=0,1,2, \ldots,
$$

where each $v_{v}(z)$ is a polynomial of degree $p-1$ at most. Then

$$
v_{v+1}(z)=[1 / g(z)]\left[v_{v}(z)-\phi_{v}(z) \Pi^{\langle p\rangle}(z)\right],
$$

where $\phi_{v}(z)$ is the polynomial such that $v_{v}(z)-\phi_{v}(z) \Pi^{\langle p\rangle}(z)$ is divisible by $g(z)$. Let

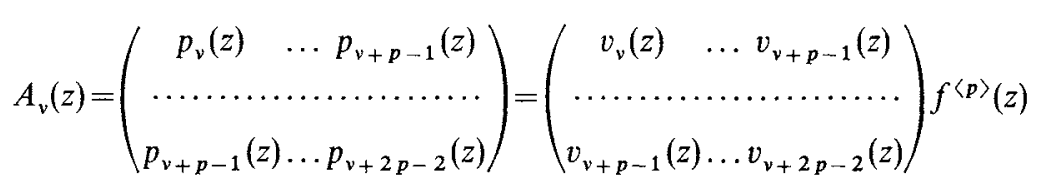

and

$$
B_{v}(z)=\left(\begin{array}{ccc}
q_{v}(z) & \ldots & q_{v+p-1}(z) \\
\ldots \ldots \ldots & \ldots & \ldots \ldots \ldots \\
q_{v+p+1} & \ldots & q_{v+2 p-2}(z)
\end{array}\right)
$$

We will consider the case when $r_{i}$ are distinct. From (3.12) we obtain

$$
v_{v}\left(r_{i}\right)=v_{0}\left(r_{i}\right) g^{-v}\left(r_{i}\right), \quad i=1,2, \ldots, p,
$$

and by Lagrangian interpolating formula

$$
v_{v}(z)=\sum_{i=1}^{p} v_{0}\left(r_{i}\right) g^{-v}\left(r_{i}\right) \Pi_{i}^{\langle p\rangle}(z) / \Pi_{i}^{\langle p\rangle}\left(r_{i}\right),
$$

where

$$
\Pi_{i}^{\langle p\rangle}(z)=\Pi^{\langle p\rangle}(z) /\left(1-r_{i}^{-1} z\right) .
$$


Hence

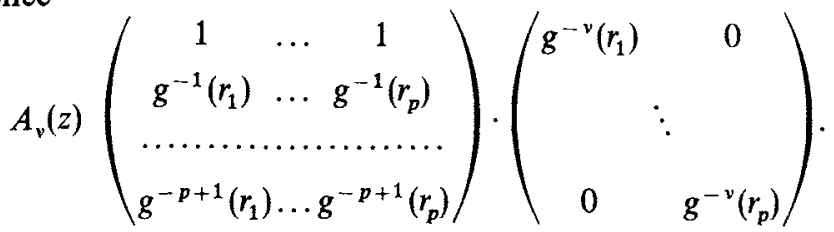

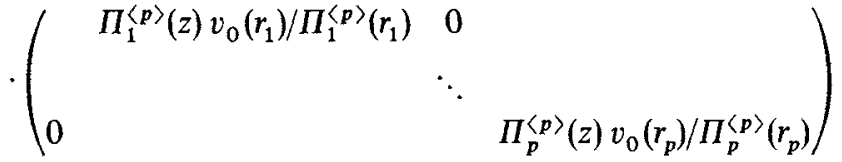

$$
\begin{aligned}
& \left(\begin{array}{cc}
1 & g^{-1}\left(r_{1}\right) \ldots g^{-p+1}\left(r_{1}\right) \\
\cdots \ldots \ldots \ldots \ldots \ldots \ldots \ldots \\
1 & g^{-1}\left(r_{p}\right) \ldots g^{-p+1}\left(r_{p}\right)
\end{array}\right) f^{\langle p\rangle}(z) .
\end{aligned}
$$

It is now easy to see from (3.9), (3.10) and (3.11) that $A_{y}^{-1}(z)$ exists for $z \in \Omega_{\rho}, z \neq r_{i}$, $i=1,2, \ldots, p$, and is given by

$$
A_{v}^{-1}(z)=A(z) G^{v} B
$$

where

$$
G=\operatorname{diag}\left[g\left(r_{1}\right), g\left(r_{2}\right), \ldots, g\left(r_{p}\right)\right],
$$

and $A(z)$ and $B$ are matrices not depending upon $v$. Confluent case can be handled in a similar way to obtain the same result.

Now, by Lemma 2.2 we know that

$$
\left\|B_{v}(z)\right\|=0\left(\rho^{-v}\right), z \in \Omega_{\rho} .
$$

Since

$$
\lim \left\|G^{v}\right\|^{1 / v}=\left|g\left(r_{p}\right)\right|
$$

for any matrix norm $\|\cdot\|[4$, p. 183], we have that, for $v$ sufficiently large,

$$
\left\|B_{v}(z) A_{v}^{-1}(z)\right\|=0\left(\left|g\left(r_{p}\right) / \rho\right|^{v}\right),
$$

with a slight adjustment of $\rho$. Since $\rho>\left|g\left(r_{p}\right)\right|$, we have that

$$
\lim _{v \rightarrow \infty}\left\|B_{v}(z) A_{\nu}^{-1}(z)\right\|=0, z \in \Omega_{\rho}, z \neq r_{i}, \quad i=1,2, \ldots, p .
$$

Moreover, for each $v$,

$$
B_{\psi}\left(r_{i}\right)=0, \quad i=1,2, \ldots, p .
$$

\section{Hence}

$$
\lim _{v \rightarrow \infty} \frac{\delta\left(A_{v}(z)+B_{v}(z)\right)}{\delta\left(A_{v}(z)\right)}=1 \quad \text { for } z \in \Omega_{\rho} .
$$


For each $v$, let

Then

$$
C_{v}(z)=\delta\left(\begin{array}{ccc}
v_{v}(z) & \ldots & v_{v+p-1}(z) \\
\ldots \ldots \ldots \ldots \ldots \ldots \ldots \ldots \\
v_{v+p-1}(z) \ldots & \ldots & v_{v+2 p-2}(z)
\end{array}\right) /\left(-I I^{\langle p\rangle}\right)^{p-1} .
$$

$$
\lim _{v \rightarrow \infty} \delta\left(A_{v}(z)\right) /[-f(z)]^{p-1}=\lim _{v \rightarrow \infty} C_{v}(z) f^{\langle p\rangle}(z) .
$$

But, Theorem 1.1, together with (3.12), gives

$$
\lim _{v \rightarrow \infty} C_{v}^{+}(z)=1 \text {. }
$$

Now, we can see from (1.7), (3.2)', and (3.13) through (3.17) that

$$
\lim _{v \rightarrow \infty} g_{v, p}^{+}(z)=f^{\langle p\rangle}(z), \quad \text { for } z \in \Omega_{\rho} .
$$

This proves the second part of the theorem. The first part of the theorem can be proved similarly using Lemma 2.3 .

\section{§4. Numerical Results}

Numerical testing has been performed on a computer program which implements the algorithm described in $\S 3$. A few comments may be in order before exhibiting numerical examples. The restrictions on the operating polynomial $g(z)$ to insure the existence of a $\rho$ satistying (3.8) for part (I) of Theorem 3.2 or (3.9) for part (II) of Theoreme 3.2 are not crucial. It is easy to see that $g(z)=z$ guarantees the existence of such a $\rho$ and it is best to start the algorithm using $g(z)=z$ if no information about the zeros of $f(z)$ is available. When the sequence $g_{v, p}^{+}(z)$, for some $p$, starts to converge, we can accelerate the convergence by using $g(z)=q_{v, p}(z)$, where $q_{v, p}(z)$ is the polynomial of degree $p$ obtained from $f(z) / g_{v, p}^{+}(z)$, since the rate of convergence depends upon the ratio $\left|g\left(r_{p+1}\right) / g\left(r_{p}\right)\right|$. In particular, if $g(z)$ is replaced by $q_{v, p}(z)$ at each step, the convergence is of order two [3]. (See Example II below.) Note that $g(z)=q_{v, p}(z)$ satisfies all the hypotheses for part (II) of Theorem 3.2.

Another advantage of this generalized algorithm is that equimodular zeros can be produced by using $g(z)=z-\alpha$, for $\alpha$ properly chosen. Numerical experiments show that a good value of $\alpha$ to be used in this case can be obtained from the sequence $g_{v}^{+}(z)$ generated using $g(z)=z$. A numerical example of this is given now.

Example I. $f(z)=\left(1-1.997512438 z+0.246268657 z^{2}+1.748756219 z^{3}\right.$

$$
\left.-1.246268657 z^{4}+0.248756219 z^{5}\right) e^{z} \text {. }
$$

zeros: $1,1,-1,2,2.01$. Table 1 contains some results of the algorithm.

Acceleration technique can be used for faster convergence in Example I but we exhibit the behavior of the acceleration method in the following example.

Example II. $f(z)=\sin z / z$; zeros: $\pm \eta \pi, \eta=1,2,3, \ldots$. 
Table 1

\begin{tabular}{|c|c|c|c|}
\hline \multirow[b]{2}{*}{$v$} & \multicolumn{3}{|l|}{$g(z)=z, g_{0}(z)=1$} \\
\hline & $q_{v, 1}(z)$ & $q_{v, 2}(z)$ & $q_{v, 3}(z)$ \\
\hline 5 & $\begin{array}{l}1-1.354569 z \\
\text { zero: } 0.7382421\end{array}$ & $1+0.02819744 z-0.9669027 z^{2}$ & $\begin{array}{l}1-1.020645 z-0.990315 z^{2} \\
\quad+1.025851 z^{3}\end{array}$ \\
\hline \multirow[t]{3}{*}{10} & $\begin{array}{l}1-1.015842 z \\
\text { zero: } 0.9844054\end{array}$ & $1-0.1983125 z-1.159201 z^{2}$ & $\begin{array}{l}1-1.001468 z-0.9993122 z^{2} \\
\quad+1.001835 z^{3}\end{array}$ \\
\hline & $\begin{array}{l}\text { [No convergence, but a } \\
\text { good approximation } \\
\text { for the first zero is } \\
\text { obtained.] }\end{array}$ & & [Convergence] \\
\hline & \multicolumn{3}{|l|}{$g(z)=z-0.7, g_{0}(z)=1$} \\
\hline$v$ & $q_{\mathrm{v}, 1}(z)$ & $q_{v, 2}(z)$ & \\
\hline 5 & $\begin{array}{l}1-1.058209 z \\
\text { zero: } 0.9449929\end{array}$ & $1-2.011597 z+1.031736 z^{2}$ & \\
\hline \multirow[t]{2}{*}{10} & $\begin{array}{l}1-1.029548 z \\
\text { zero: } 0.9713000\end{array}$ & $1-2.000000 z+0.9999965 z^{2}$ & \\
\hline & [Slow convergence] & [Convergence] & \\
\hline
\end{tabular}

Remark. $q_{v, 2}(z)$ converges to the factor $q(z)=(1-z)^{2}$ of $f(z)$. Any polynomial with one zero of integer multiplicity can be solved easily. In this case $q(z)$ is quadratic and we can use quadratic formula.

\begin{tabular}{|c|c|}
\hline Replace & $\begin{array}{l}f(z) \text { by } f(z) / q(z) \\
g(z)=z, g_{0}(z)=1 \\
q_{y}(z)\end{array}$ \\
\hline 5 & $\begin{array}{l}1+1.015636 z \\
\text { zero: }-0.984604453\end{array}$ \\
\hline 10 & $\begin{array}{l}1+0.9992598 z \\
\text { zero: }-1.0007407065 \\
\text { [Convergence] }\end{array}$ \\
\hline
\end{tabular}

Remark. The other two zeros can also be obtained similarly.

\section{Table 2}

\begin{tabular}{rll}
\hline$v$ & $g(z)=z$ & Varying $g(z)$ from $v=5$ \\
\hline 1 & \pm 2.927700219 & \\
2 & \pm 2.927700219 & \\
3 & \pm 3.079589415 & \\
4 & \pm 3.079589415 & \\
5 & \pm 3.124704710 & \pm 3.141000248 \\
6 & \pm 3.124704710 & \pm 3.141592306 \\
7 & \pm 3.137176863 & \pm 3.141592654 \\
8 & \pm 3.137176863 & {$[$ Convergence] } \\
9 & \pm 3.140464067 & \\
30 & \pm 3.141592652 & \\
& {$[$ Convergence] } & \\
\hline
\end{tabular}


The roots computed from each $q_{v, 2}(z)$ in the original method with $g(z)=z$ and in the accelerated method are given in Table 2.

Acknowledgement. The author wishes to express her sincere gratitude to Dr. Alston S. Householder for his valuable suggestions and help for the preparation of this paper, and to the referee for many helpful comments.

\section{References}

1. Bauer, L.: Beiträge zur Entwicklung numerischer Verfahren für programmgesteuerte Rechenanlagen. I. Quadratisch konvergente Durchfürung der Bernoulli-Jacobischen Methoden zur Nullstellenbestimmung von Polynomen. Bayer. Akad. Wiss. Math. nat. K1. S.-B., 275-303 (1954)

2. Bauer, F.L.: Beiträge zur Entwicklung numerischer Verfahren für programmgesteuerte Rechenanlagen. Il. Direkte Faktorisierung eines Polynoms. Bayer. Akad, Wiss. Math. K1. S.-B., 163-203 (1956)

3. Chung, S.P.: Generalization and Acceleration of an Algorithm of Sebastião e Silva and Its Duals. Numer. Math. 25, 365-377 (1976)

4. Householder, A.S.: The Theory of Matrices in Numerical Analysis. Blaisdell Publishing Company, New York, 1964

5. Householder, A.S.: Generalizations of an Algorithm of Sebastião e Silva. Numer. Math. 16, 375-382 (1971)

6. Householder, A.S.: Multigradients and the Zeros of Transcendental Functions. Linear Algebra and Its Application, 4, 175-182 (1971)

7. Sebastião e Silva, J.: Sur une méthode d'approximation semblable a celle de Graeffe. Portugal Math. 2, 271-279 (1941)

8. Stewart, G.W.: On a Companion Operator for Analytic Functions. Numer. Math. 18, 26-43 (1971)

9. Walsh, J.L.: Interpolation and Approximation by Rational Functions in the Complex Domain. American Mathematical Society Colloquium Publications, Volume XX, New York, 1935 